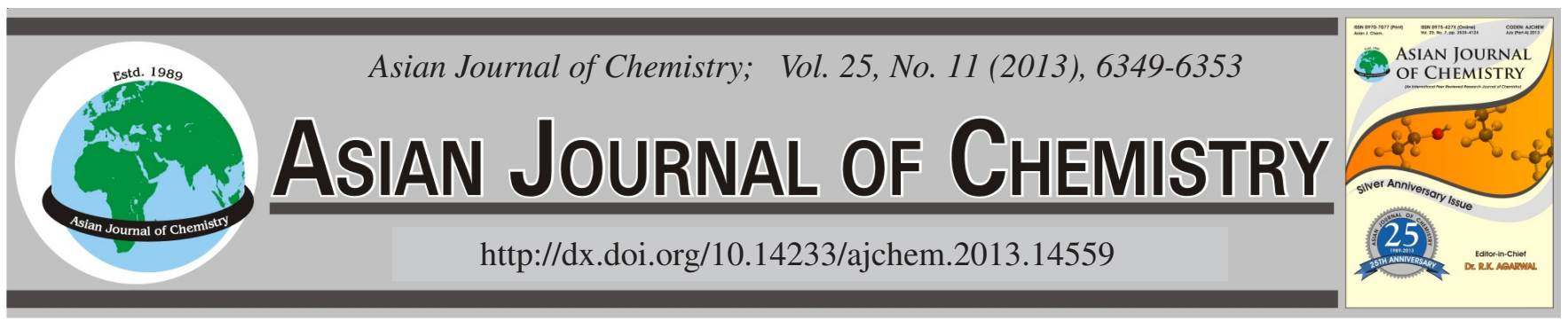

\title{
Preparation and Antifouling Properties of Poly(vinyl alcohol)-co- Poly(acrylamide) Macroporous Hydrogels
}

\author{
Weiwei Cong*, Ling Xiao, Ke Wang, Zhengtao Chen, Xueyan Yu and Taijiang Gui
}

Marine Chemical Research Institute Co. Ltd, State Key Laboratory of Marine Coatings, Qingdao, P.R. China

*Corresponding author: Tel: +86 532 85845879; E-mail: congweiwei19830211@163.com

\begin{abstract}
In recent years, high-efficiency and non-toxic antifouling techniques are predominant in view of environmental protection and public safety. In this paper, biomimetic antifouling technique was carried out. Poly(vinyl alcohol)-co-poly(acrylamide) (PVA/PAM) macroporous hydrogels were synthesized by porogenic technique using PEG200, PEG1000 and PEG6000 as pore forming agents, polyvinyl alcohol and acrylamide (AM) as raw materials and while polyvinyl alcohol could enhance the mechanical strength of macroporous hydrogels. Poly(ethylene glycol) with different molecular weight made different pore structure, when macroporous hydrogel had a porous structure with a pore size mostly between 10-30 $\mu \mathrm{m}$ with PEG1000 and possessed good mechanical strength which was suitable for the culture medium of marine bacterium. Further, we extracted Kytococcus sedentarius which had bacteriostasic activity from marine microbial biofilm. PVA/PAM macroporous hydrogel with Kytococcus sedentarius in it made biomimetic biofilm and their antifouling performance were tested in the sea for 5 months. It is suggested that the biomimetic biofilm with only a little green alga on it for 5 months demonstrated better antifouling properties.
\end{abstract}

Key Words: Macroporous hydrogel, Kytococcus sedentarius, Biofilm, Antifouling properties.

\section{INTRODUCTION}

All structures immersed in seawater coverd a layer which called conditioning film ${ }^{1-3}$ in several min and then bacterium, algae and their spores attached to it to form microbial biofilm ${ }^{46}$. Microbial biofilm is the first stage of the attachment of marine organisms. Microorganisms living in microbial biofilm play an important role in the attachment of macrofouling, both in terms of its facilitation and its inhibition. Some microorganisms secrete extracellular polymeric substances ${ }^{7-10}$ (known as exopolysaccharide, are high-molecular weight compounds) which can inhibit the adhesion of marine organisms. And these microorganisms and their metabolites are environment-friendly which have received much attention in antifouling field.

Mary et al. ${ }^{11}$ isolated 16 marine bacteria from microbial biofilm of Balanus amphitrete, 12 of them could restrict the attachment of Balanus reticulates larvae. Biofilms of the bacterium, Pseudoalteromonas tunicata, extracted from the surface of a tunicate, showed antifouling activity against Balanus amphrite and Ciona intestinalis larvae ${ }^{12,13}$. Burgess et al. ${ }^{14}$ reported the development of an antifouling coating incorporating extracts from a marine bacterium as the active principal. The coating exhibited good activity against marine bacteria, barnacle larvae and algal spores, which suggests potential use as an antifouling paint.
Macroporous hydrogels ${ }^{15-17}$ are a special class of porous hydrophilic water-insoluble polymers that can swell in water and hold a huge amount of water while maintaining their structure even under pressure. Open pores of macroporous hydrogels are the channel of water pervasion. The pore structures can form capillary channels, water is taken up into dried hydrogels by capillary rise, in addition, pore structure increases surface area contacting with water and quicken water pervasion into macroporous hydrogels. So macroporous structure infinitely expands the application of macroporous hydrogels. Because of their excellent properties they are utilized in agricultural, horticultural, biomedical and pharmaceutical applications. They can also be used for cell culture. The desired properties of macroporous hydrogels are high swelling capacity, high swelling rate and good mechanical strength of the swollen gel. Recently, porous superabsorbent hydrogels have gained much attention because of their improved swelling and absorption rate.

In this context, we synthesized macroporous hydrogels with finer mechanical strength, compared the effect of pore structure with different molecular weight poly(ethylene glycol) and extracted Kytococcus sedentarius from marine biofilm which was cultured in macroporous hydrogels to make biomimetic biofilm. This new technique enriched antifouling field. 


\section{EXPERIMENTAL}

Acrylamide (AM) monomer and N,N'-methylene bis acrylamide (MBA) cross-linker which is water-soluble, supplied by Aladdin, were used without further purification. Ammonium persulfate (APS), poly(vinyl alcohol) (PVA), poly(ethylene glycol) 200 (PEG200), poly(ethylene glycol) 1000 (PEG1000), poly(ethylene glycol) 6000 (PEG6000) and $\mathrm{N}, \mathrm{N}, \mathrm{N}^{\prime}, \mathrm{N}^{\prime}$-tetramethylethylenediamide (TMEDA) all from Aladdin were used as received.

There are four bacteria of Vibrio anguillarum (gram-negative bacteria), Bacillus cereus (gram-positive bacteria), Pseusomonas aeruginosa (gram-negative bacteria) and Pseudomonas putida (gram-negative bacteria) provided by the Lab of microorganism in the Ocean University of China. They were cultivated in the biology laboratory and prepared for the experiment.

Preparation of poly(vinyl alcohol)/poly(acrylamide) macroporous hydrogels ${ }^{18}$ : Macroporous hydrogels were synthesized by free-radical polymerization of acrylamide, poly(vinyl alcohol) and crosslinker $\mathrm{N}, \mathrm{N}^{\prime}$-methylene bis acrylamide with an ammonium persulfate/N,N,N',N'tetramethylethylenediamide redox pair initiating system in the presence of pore-generator (poly(ethylene glycol)). In a typical reaction, $14.07 \mathrm{mM}$ acrylamide, $0.32 \mathrm{mM} \mathrm{N}, \mathrm{N}^{\prime}$-methylene bis acrylamide and $2.5 \mathrm{~g}$ PEG200 were dissolved in $15 \mathrm{~mL}$ distilled water. Then the total reaction mixture was transferred to $10 \%$ (mass ratio) polyvinyl alcohol (poly(vinyl alcohol) solution prepared in advance) solution and $0.22 \mathrm{mM}$ ammonium persulfate and $0.05 \mathrm{~mL} \mathrm{~N}, \mathrm{~N}, \mathrm{~N}^{\prime}, \mathrm{N}^{\prime}$-tetramethylethylenediamide were added sequentially to the reaction mixture and the solution was well mixed with a electromagnetic stirrer at $30{ }^{\circ} \mathrm{C}$ and reacted for $2 \mathrm{~h}$, after that, the mixture were poured into a watch glass $20 \mathrm{~cm}$ in diameter for $3-5 \mathrm{~mm}$. The watch glass was $-20^{\circ} \mathrm{C}$ freezed for $24 \mathrm{~h}$. The reactions were carried out by varying the reaction parameters such as different molecular weight of poly(ethylene glycol). The synthesized poly(vinyl alcohol)/ poly(acrylamide) macroporous hydrogels were transferred into a $500 \mathrm{~mL}$ beaker containing distilled water and left for $24 \mathrm{~h}$ while changing the water every $2 \mathrm{~h}$ in order to remove the porogenic agent and other unreacted monomers.

IR spectra of poly(vinyl alcohol)/poly(acrylamide) macroporous hydrogels: Take poly(vinyl alcohol)/poly(acrylamide) macroporous hydrogel with PEG1000 as pore-forming agent for example. The macroporous hydrogel was on the vacuum drying condition and then milled a shape on thin sheet with $\mathrm{KBr}$ and carried out on AVATAR-380 spectrophotometer (Thermo Nicolet Company, USA).

Characterization of poly(vinyl alcohol)/poly(acrylamide) macroporous hydrogels: Macroporous hydrogels with equilibrium water content were wiped away exterior water with filter paper and cut into $1 \mathrm{~cm}^{3}$ cubes for observation. The structural and morphological variations were observed by using three dimensional digital microscopes (HIROX, KH-7700, Questar China Limited).
Mechanical strength of poly(vinyl alcohol)/poly(acrylamide) macroporous hydrogels ${ }^{19,20}$ : A bench comparator (Fig. 1) was used to test the mechanical properties of the macroporous hydrogels. A gauge was fastened to the bracket and a lab jack was on the bottom, the sample swollen in 2216E marine bacterium liquid nutrient medium $(0.10 \%(\mathrm{w} / \mathrm{v})$ yeast power, $0.5 \%(\mathrm{w} / \mathrm{v})$ peptone, seawater, $115^{\circ} \mathrm{C}$ sterilization 0.5 h) was put on the lab jack, above the sample, there was a thin aluminum foil film. The bench comparator was adjusted to make the sample ascend slowly. The hydrogel was compressed by applying increasing amounts of weights until a point when the macroporous hydrogels started cracking, the pressure at that point was defined as ultimate compression pressure $(\mathrm{Fu} / \mathrm{N})$ and the touch area of the aluminum foil film was defined as contact area $\left(\mathrm{S} / \mathrm{cm}^{2}\right)$. So intensity of pressure $(\mathrm{P} / \mathrm{KPa})$ of macroporous hydrogels was determined to characterize the mechanical properties of the poly(vinyl alcohol)/poly(acrylamide) macroporous hydrogels as Scheme-I.

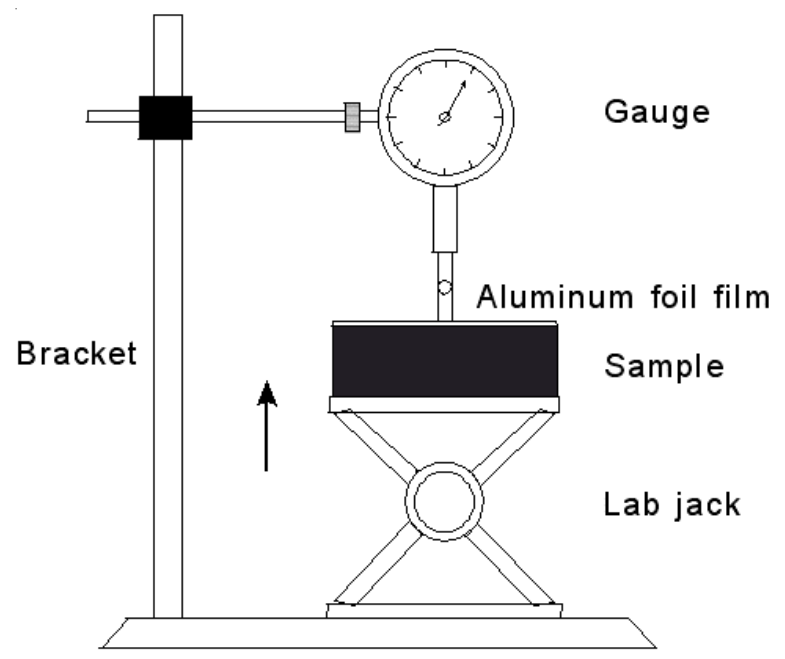

Fig. 1. A bench comparator with modifications used to examine the mechanical property of macroporous hydrogels

$$
\mathrm{P}=\frac{\mathrm{Fu}}{\mathrm{S}}
$$

Scheme-I: Formula of intensity of pressure

Antifouling properties of poly(vinyl alcohol)/poly(acrylamide) macroporous hydrogels ${ }^{21-23}$ : Marine biofilm was collected from the board with antifouling coating in Qingdao Port, cultivated in 2216E liquid nutrient medium. And one marine bacterium of Kytococcus sedentarius was extracted and purified from marine biofilm. Bacteriostatic circle test proved that Kytococcus sedentarius could inhibit Vibrio anguillarum and Bacillus cereus, but had little effect on Pseusomonas aeruginosa and Pseudomonas putida (Table-1).

Poly(vinyl alcohol)/poly(acrylamide) macroporous hydrogel with pore-generator of PEG1000 swelled in 2216E

\begin{tabular}{cccc} 
& \multicolumn{4}{c}{ TABLE-1 } \\
& \multicolumn{2}{c}{ BACTERIOSTATIC ACTIVITY OF Kytococcus sedentarius } \\
\hline \multirow{2}{*}{ Extracted bacterium } & & \multicolumn{2}{c}{ Tested bacteria } \\
\cline { 2 - 4 } & Vibrio anguillarum & Bacillus cereus & Pseusomonas aeruginosa \\
\hline Kytococcus sedentarius & ++ & ++ & Pseudomonas putida \\
\hline
\end{tabular}


liquid nutrient medium of Kytococcus sedentarius which made the biomimetic biofilm, the biomimetic biofilm was then fastened to the board and immersed in the sea to a depth of $1 \mathrm{~m}$ for 5 months.

\section{RESULTS AND DISCUSSION}

Synthesis of poly(vinyl alcohol)/poly(acrylamide) macroporous hydrogels: Poly(vinyl alcohol) is a water-soluble wearable macromolecule polymer which has good tensile strength and impact resistance, so it is a fine additive to improve the mechanical strength of macroporous hydrogels ${ }^{24-26}$. During the process of forming hydrogel, hydrogen bond of intrachain hydroxyl in poly(vinyl alcohol) molecule associated to twine, similar to point of crosslinked network structure. Three-dimensional network structure of self-crosslinking poly(vinyl alcohol) contributes a lot to the mechanical strength of macroporous hydrogels ${ }^{27}$. In the ammonium persulfate/ $\mathrm{N}, \mathrm{N}, \mathrm{N}^{\prime}, \mathrm{N}^{\prime}$-tetramethylethylenediamide redox pair initiating system, hydrogen atom of hydroxyl was ionized, produced much point of crosslinked of poly(vinyl alcohol) and hydrogen atom of poly(vinyl alcohol) molecule was easily excitated by $\mathrm{S}_{2} \mathrm{O}_{3}{ }^{2-}$ of ammonium persulfate ${ }^{28,29}$. Some poly(vinyl alcohol) molecule copolymerized with prepolymers of poly(acrylamide), others formed three-dimensional network structure by selfcrosslinking and this spatial structure contributed a lot to the mechanical strength of macroporous hydrogels. The hydrogels were freezed for $24 \mathrm{~h}$ at $-20{ }^{\circ} \mathrm{C}^{30-34}$ and this physical crosslinking method made the structure of hydrogels more compact.

IR spectra of poly(vinyl alcohol)/poly(acrylamide) macroporous hydrogels: The IR spectra of poly(vinyl alcohol)/poly(acrylamide) macroporous hydrogels showed peaks corresponding to the functional groups of the monomers in the polymeric chains. The observed peaks at $3409 \mathrm{~cm}^{-1}$, corresponding to hydrogen-bonded (bridged) N-H stretching because of acrylamide and N,N'-methylene bis acrylamide; $2907 \mathrm{~cm}^{-1}$, corresponding to the saturated $\mathrm{C}-\mathrm{H}$ group of main chain of polymer; $1665 \mathrm{~cm}^{-1}$, corresponding to the $\mathrm{C}=\mathrm{O}$ of acrylamide and N,N'-methylene bis acrylamide; $1103 \mathrm{~cm}^{-1}$, corresponding to the $\mathrm{C}-\mathrm{O}$ group of the poly(vinyl alcohol) unit. But the C-O-C group was not found at $1050-1150 \mathrm{~cm}^{-1}$, which showed PEG1000 was only used as pore-forming agent in the preparation of macroporous hydrogel and it did not join the reaction and was washed away after the polymerization. From the IR analysis, it was clearly confirmed that acrylamide, poly(vinyl alcohol) and N,N'-methylene bis acrylamide units were incorporated into the copolymer chain.

Morphology of poly(vinyl alcohol)/poly(acrylamide) macroporous hydrogels: The morphology structure of poly(vinyl alcohol)/poly(acrylamide) macroporous hydrogels were studied by three dimensional digital microscope $(\times 350$ $\times 3500$ ). The micrograph of the hydrogels prepared in different pore-forming agents given in Fig. 2.

Presented in Fig. 2(a), showed no porosity and the surface of hydrogel was almost flat and compact. In contrast, the three dimensional digital microscope micrographs of the hydrogel prepared in the same concentrations of pore-forming agents, given in Fig. 2(b), showed many pores with the diameter between 10-30 $\mu \mathrm{m}$, mostly were $10 \mu \mathrm{m}$. And in Fig. 2(c), it

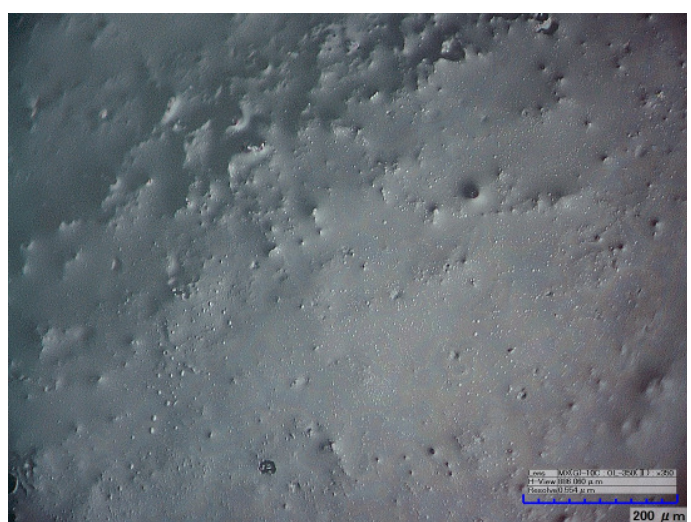

(a)

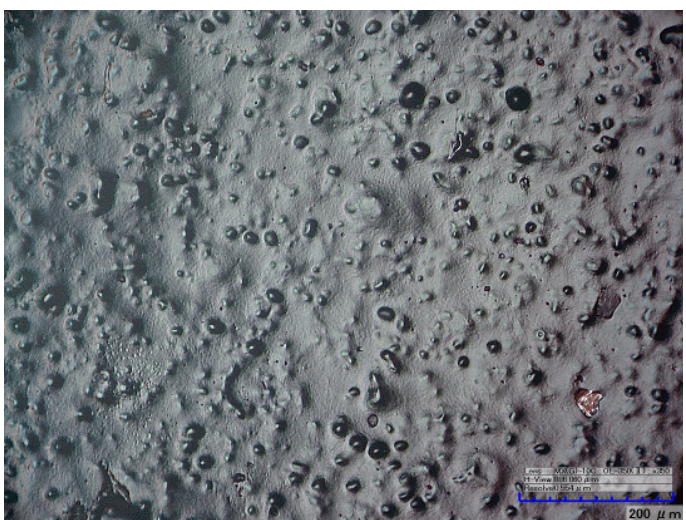

(b)

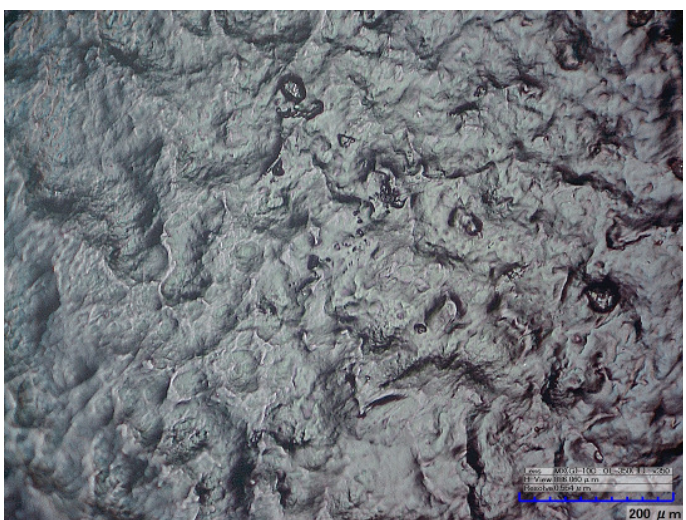

(c)

Fig. 2. Micrographs of poly(vinyl alcohol)/poly(acrylamide) macroporous hydrogels prepared with (a) $6 \%$ (wt) PEG200 (b) $6 \%$ (wt) PEG1000 and (c) $6 \%$ (wt) PEG6000

had the same amount of PEG6000, showed pore channels and layers instead of pores. Further, different molecular weight of poly(ethylene glycol) had a synergistic effect on the obtaining of pore structure. Thus, the number of pore structure increased with an increase in the molecular weight of poly(ethylene glycol) used for the polymerization reactions and when the molecular weight of poly(ethylene glycol) was too big, for instance PEG 6000, pore channels instead of pore structure and there was no pore structure.

Mechanical strength of poly(vinyl alcohol)/poly(acrylamide) macroporous hydrogels: One of the most important requirements for a biomimetic biofilm of macroporous hydrogel is its structural integrity. A macroporous hydrogel 
should be able to withstand the pressure in the sea during checking its antifouling property. Formulation variables, such as the concentration of poly(vinyl alcohol), amount of crosslinker, type of monomer and pore-forming agents, all affect the mechanical properties of the macroporous hydrogels. In this study, we examined the effect of the concentrations of poly(vinyl alcohol) on the mechanical strength of macroporous hydrogel composites.

Poly(vinyl alcohol)/poly(acrylamide) macroporous hydrogels containing different concentrations of poly(vinyl alcohol) (wt 1-20\% of distilled water, preparation in advance) were obtained. The mechanical properties of these macroporous hydrogels were studied on a bench comparator after they swelled to equilibrium size in $1 \mathrm{~cm} \times 1 \mathrm{~cm} \times 1 \mathrm{~cm}$. As shown in Fig. 3, the incorporation of poly(vinyl alcohol) contributed to the mechanical stress of hydrogels. It decreased the swelling size of the macroporous hydrogels under pressure. On the other hand, it considerably increases the fracture stress value of macroporous hydrogels with the increased of poly(vinyl alcohol) when the concentration of poly(vinyl alcohol) was less than $10 \%$, while from $10-20 \%$ the increase of mechanical property was not obvious. A poly(vinyl alcohol) concentration of $10 \%$ was found to be the best among the conditions studied for the improvement of the mechanical strength. And the fracture stress of macroporous hydrogels was $2.32 \mathrm{MP}$ with poly(vinyl alcohol) concentration of $10 \%$. The possible reason for the improved structural integrity is that the addition of poly(vinyl alcohol) increases the physical cross-linking density of the

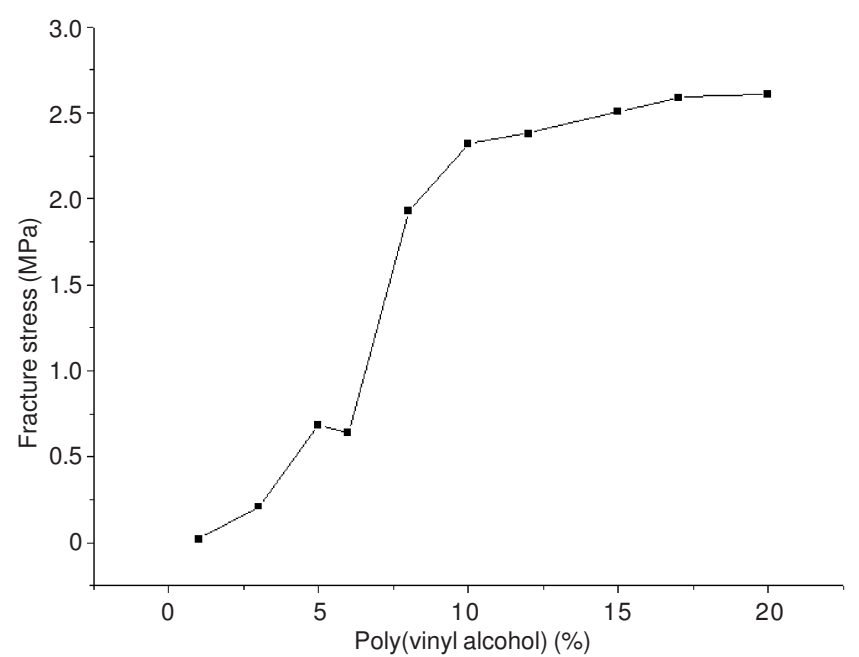

Fig. 3. Fracture stress of macroporous hydrogels with different concentrations of poly(vinyl alcohol) macroporous hydrogels. However, if too much poly(vinyl alcohol) is incorporated, due to the increase of solution viscosity, good mixing of all the ingredients becomes difficult and it contributes little to the mechanical stress of macroporous hydrogels which increases their cost. In most of the studies, $10 \%$ poly(vinyl alcohol) was used.

Result of test board in the sea: Being obvious from the study of anti-bacterial capability, Kytococcus sedentarius had good inhibition properties and then we put it into poly(vinyl alcohol)/poly(acrylamide) macroporous hydrogel to make biomimetic biofilm, the experiment was conducted in the sea for 150 days from June to October in 2011 when entermorpha and barnacle represented vigorous growth in this period. The results were shown in Table-2.

The formation of biofilm is the first stage of biofouling. Control of the first stage effectively could inhibit the attachment of other algae and macrofouling organisms. Based on photographic records, we investigated antifouling property of biomimetic biofilm from the surface-attaching marine organisms. From Table-2, there was nothing on the surface of biomimetic biofilm for 30 days and only a little silt in the middle of it for 2 months. Until 90 days, in the middle of biomimetic biofilm, there were some green algae. Up to 120 days, the green algae took one third area of the biomimetic biofilm and a little silt was on the edge of it. The edge was full of silt and the green algae increased a lot on the surface of biomimetic biofilm for 150 days. From the figures above, we could see clearly biomimetic biofilm with Kytococcus sedentarius have good antifouling performance especially at the most vigorous period of barnacle. And this biomimetic technique could not pollute the entironment, so it has a good application prospect in antifouling coating.

\section{Conclusion}

Macroporous hydrogels consisting of polyvinyl alcohol and acrylamide crosslinked with N,N'-methylene bisacrylamide were found to be hydrophilic. The spatial structure and mechanical stress of them were greatly influenced by the chemical architecture of the hydrogel network structure. The effect of different molecular weight of poly(ethylene glycol) on the apparent structure was investigated in detail and the results indicated that macroporous hydrogel with PEG1000 could get pore size mostly between 10-30 $\mu \mathrm{m}$ and also the concentration of poly(vinyl alcohol) solution was checked their mechanical strength which showed $10 \%$ poly(vinyl alcohol) solution contributed the most. Biomimetic biofilm with poly(vinyl alcohol)/poly(acrylamide) macroporous

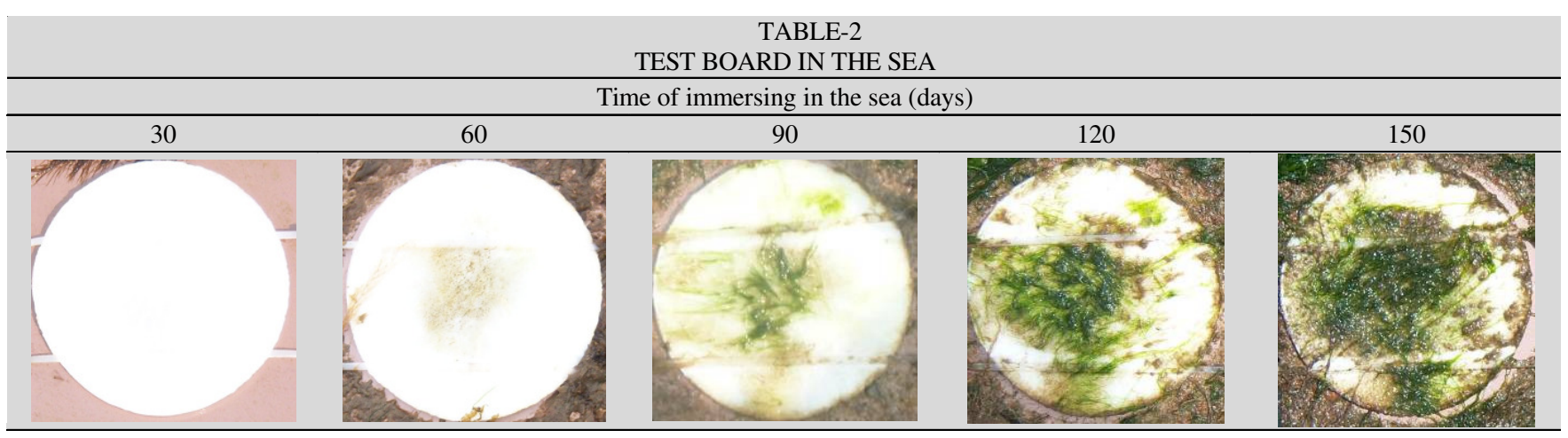


hydrogel showed good inhibition property to marine organisms for 150 days which promoted the development of environmentally friendly antifouling technology.

\section{REFERENCES}

1. B.K. Canales, L. Higgins, T. Markowski, L. Anderson, Q.A. Li and M. Monga, J. Endourol., 23, 1437 (2009).

2. A. Garg, A. Jain and N.B. Bhosle, Int. Biodeter. Biodegrad., 63, 7 (2009).

3. M. Gabi, L. Hefermehl, D. Lukic, R. Zahn, J. Vörös and D. Eberli, Urol. Res., 39, 81 (2011).

4. J.W. Costerton, Z. Lewandowski, D.E. Caldwell, D.R. Korber and H.M. Lappin-Scott, Ann. Rev. Microbiol., 49, 711 (1995).

5. R.Amann, J. Snaidr, M. Wagner, W. Ludwig and K.H. Schleifer, J. Bacteriol., 178, 3496 (1996).

6. H. Anwar, J.L. Starp and W.J. Costerton, Antimicrob. Agents. Chemother., 36, 1347 (1992).

7. J.N. Yan, Y.B. Xu, X.J. Duan and Y.Y. Du, Sci. Technol., 27, 106 (2009).

8. I. Dogsa, M. Kriechbaum, D. Stopar and P. Laggner, Biophys. J., 89 2711 (2005)

9. H. Liu and H.H. Fang, J. Biotechnol., 95, 249 (2002).

10. W.M. Zeng, G.Z. Qiu, H.B. Zhou, X.D. Liu, M. Chen, W.L. Chao, C.G. Zhang and J.H. Peng, Hydrometallurgy, 100, 177 (2010).

11. Sr. A. Mary, Sr. V. Mary, D. Rittschof and R. Nagabhushanam, J. Chem. Ecol., 19, 2155 (1993)

12. C. Holmstrom and S. Kjelleberg, FEMS Microbiol. Ecol., 30, 285 (1999).

13. S.G. James, C. Holmstrom and S. Kjelleberg, Appl. Environ. Microbiol., 62, 2783 (1996)

14. J.G. Burgess, K.G. Boyd, E. Armstrong, Z. Jiang, L.M. Yan, M. Berggren, U. May, T. Pisacane, A.K. Granmo and D.R. Adams, Biofouling, 19S, 197 (2003).

15. F. Topuz and O. Okay, React. Funct. Polym., 69, 273 (2009).
16. T. Caykara, M. Bulut, N. Dilsiz and Y. Akyüz, J. Macromol. Sci., Phys. A: Pure Appl. Chem., 43, 889 (2006).

17. A.G. Lee, C.P. Arena, D.J. Beebe and S.P. Palecek, Biomacromolecules, 11, 3316 (2010).

18. T.D. Dziubla and A.M. Lowman, J. Biomed. Mater. Res. A, 68, 603, (2004).

19. H. Omidian, K. Park and P. Sinko, Pharmaceutical Polymers In: Martin's Physical Pharmacy and Pharmaceutical Sciences, Lippincott Williams \& Wilkins, Ch. 20, pp. 492-515 (2010).

20. G. Mun, I. Suleimenov, K. Park and H. Omidian, Superabsorbent Hydrogels in Biomedical Applications of Hydrogels Handbook, Springer, New York, Ch. 20, p. 375 (2010).

21. Y. Qian, A. Nadia, T. Falk and U. Mathias, J. Mater. Chem., 21, 2783 (2011).

22. Y.M. Mohan, P.S. Keshava Murthy and K.M. Raju, J. Appl. Polym. Sci., 101, 3202 (2006).

23. J. Texter, Colloid Polym. Sci., 287, 313 (2009).

24. I.R. Rodrigues, M.M. de Camargo Forte, D.S. Azambuja and K.R.L. Castagno, React. Funct. Polym., 67, 708 (2007).

25. Y.F. Tang, Y.M. Du, X.W. Hu, X.-W. Shi and J.F. Kennedy, Carbohydr. Polym., 67, 491 (2007).

26. C.M. Xiao and M.L. Yang, Carbohydr. Polym., 64, 37 (2006).

27. A.L. Kjoniksen and B. Nystrom, Macromolecules, 29, 5215 (1996).

28. N. Kim, E.D. Sudol and V.L. Dimonie and M.S. El-Aasser, Macromolecules, 37, 2427 (2004).

29. N. Kim, E.D. Sudol, V.L. Dimonie and M.S. El-Aasser, Macromolecules, 36, 5573 (2003).

30. X.Z. Zhang and R.X. Zhuo, Macromol. Chem. Phys., 200, 2602 (1999).

31. W. Xue, S. Champ, M.B. Huglin and T.G.J Jones, Eur. Polym. J., 40, 703 (2004).

32. V. Lozinsky, Russ. Chem. Bull., 57, 1015 (2008).

33. M.V. Dinu, M.M. Ozmen, E.S. Dragan and O. Okay, Polymer, 48, 195 (2007).

34. S. Jin, F. Bian, M. Liu, S, Chen and H.L. Liu, Polym. Int., 58, 142 (2009). 